community-based education programmes focusing on changes to sexual behaviour. However, STIs rates are still high, and the problem needs more concrete and sustained efforts for its control. Screening for Ct., Ng. and Uu. is recommended during pregnancy. Based on our finding in this study, the overall high incidence of $\mathrm{Ct}$. and Uu. support screening recommendations for pregnant women in Sabzevar- Iran.

\section{P3.273 MOLECULAR TYPING AND DETECTION OF MACROLIDE RESISTANCE MUTATIONS IN T. PALLIDUM STRAINS FROM CALI, COLOMBIA}

doi:10.1136/sextrans-2013-051184.0729

'A R Cruz, ${ }^{2} \mathrm{~A}$ Pillay, ${ }^{1} \mathrm{~L}$ G Ramirez, ${ }^{2} \mathrm{H}$ Chi, ${ }^{1} \mathrm{C}$ Valencia, ${ }^{1} \mathrm{~N}$ Alexander, ${ }^{3,4,1} \mathrm{~J}$ Radolf, $3,4,1 \mathrm{~J} \mathrm{C}$ Salazar. ' CIDEIM, Cali, Colombia; ${ }^{2}$ Centers for Disease Control and Prevention, Atlanta, GA, United States; ' ${ }^{3}$ niversity of Connecticut Health Center, Farmignton, CT, United States; ${ }^{4}$ Connecticut Children's Medical Center, Hartford, CT, United States

Background Cali is a syphilis-endemic area, with a very high prevalence of gestational syphilis (14.7/1000 live births) and congenital syphilis (7.7/1000 live births). Molecular typing techniques are useful for studying bacterial strain diversity, molecular epidemiologic patterns and antimicrobial resistance patterns. The study objective was to determine the T. pallidum (Tp) strain diversity and analyse for the mutations associated with macrolide-resistance in this syphilis-endemic region.

Methods 19 secondary syphilis (SS) patients, 15 (RPR+, FTAABS +, HIV-) and 4 (RPR +, FTA-ABS +, HIV+) were enrolled in the study. Tp DNA was extracted from syphilis skin lesions and quantified by real-time-qPCR targeting polA gene. Molecular typing was performed using established typing and subtyping methods (arp, tpr, tp0279, tp0548) and strains were analysed for A2058G and A2059G mutations within the 23S rRNA gene. Strain diversity in Cali strains (arp and tpr) was compared with other published Tp molecular studies using the Shannon index.

Results 14/19 SS patients were polA PCR positive (range 5.4 38.920; mean 3.227 copies/ug total DNA). Among 8/14 samples that were fully typed, 7 strain subtypes $(21 a 11 / d, 12 d 9 / \mathrm{f}, 10 \mathrm{p} 9 / \mathrm{f}$, 14j10f, $5 p 9 / \mathrm{f}, 14 k 9 / \mathrm{f}, 14 d 9 / \mathrm{f})$ were identified with only 2 strains exhibiting identical patterns (21a11/d). The A2059G mutation was found in 1 specimen from an HIV-negative subject. Cali Tp strain types had a Shannon index of 2.2, higher than all 13 studies in other localities reported in two recent reviews.

Conclusions There is a high T. pallidum strain diversity in Cali, Colombia, probably due to population mobilisation and close proximity to other syphilis-endemic regions (i.e. Buenaventura, Pacific Coast). Contact tracing and cluster identification is difficult to achieve in this setting. Although our sample size was small, the identification of the A2059G mutation suggests that macrolides should be used with caution for syphilis treatment in Cali and close monitoring for macrolide resistant strains should be initiated.

\section{P3.274 CHARACTERIZATION OF VAGINAL LACTOBACILLUS AMONG NON-PREGNANT WOMEN WITH AND WITHOUT BACTERIAL VAGINOSIS IN INDIA AND US}

doi:10.1136/sextrans-2013-051184.0730

\begin{abstract}
.2P Madhivanan, ${ }^{1} \mathrm{~A}$ Rumphs, ${ }^{2} \mathrm{~K}$ Ravi, ${ }^{3} \mathrm{E}$ Raphael, ${ }^{2} \mathrm{~A}$ Arun, ${ }^{2} \mathrm{~V}$ Srinivas, ${ }^{4} \mathrm{~J} \mathrm{D}$ Klausner, ${ }^{3} \mathrm{~L}$ W Riley, ${ }^{2,1} \mathrm{~K}$ Krupp. 'Robert Stempel College of Public Health \& Social Work, Florida International University, Miami, FL, United States; ${ }^{2}$ Public Health Research Institute of India, Mysore, India; '3School of Public Health, University of California Berkeley, Berkeley, CA, United States; ${ }^{4}$ Geffen School of Medicine, University of California, Los Angeles, Los Angeles, CA, United States
\end{abstract}

Background Lactobacillus is an integral part of vaginal microbiota that maintains healthy environment and plays an important role in preventing sexually transmitted infections and HIV. We profile the Lactobacillus species present when the women are healthy or have Bacterial Vaginosis among women in US and India.

Method Between February 2010 and November 2011, a sample of 75 women attending Prerana Women's Health Clinic in India or San Francisco City Clinic in USA were sampled and diagnosed for BV based on Amsel's Criteria. In addition, Gram stained smears of vaginal fluid were Nugent scored. Vaginal swabs were then cultured in MRS broth. Gram positive Lactobacilli generating about $600-800 \mathrm{bp}$ amplicon by $16 \mathrm{SrDNA}$ PCR with $16 \mathrm{~S}$ primers were further characterised by sequencing.

Result Vaginal samples were obtained from 75 women. According to Amsel criteria, 34 women were healthy and 41 women had BV. Lactobacilli were isolated from 22 healthy Indian and 10 healthy US women. Lactobacilli were also isolated from 4 Indian and 5 US women with BV. Eleven Lactobacillus species were isolated from 26 Indian women and 9 species were identified from 15 US women. The common Lactobacilli species found in Indian women included L. Crispatus (24.3\%), L. Gasseri (24.3\%), and L. Jensenii $(13.5 \%)$, while L. Crispatus (32.0\%), L. Jensenii (20.0\%), and L. Coleohominis $(12.0 \%)$ were common in US women. L. crispatus was cultured from $44 \%$ of healthy and $4.9 \%$ of women with BV. L. jensenii, $L$. gasseri, and L. acidophilus were cultured from $25.6 \%, 23.5 \%$ and $2.9 \%$ of healthy women; and $2.4 \%, 4.9 \%$ and $0.0 \%$ of BV women, respectively.

Conclusion Our findings showed lactobacilli species present in healthy vagina of women in India do not differ from those reported from other countries. This information is useful to development of microbicides for HIV prevention.

\section{P3.275 NEISSERIA GONORRHOEAE: SITUATION OF ANTIBIOTIC RESISTANCE IN GERMANY}

doi:10.1136/sextrans-2013-051184.0731

S Buder, P K Kohl. Konsiliarlabor für Gonokokken, Klinik für Dermatologie und Venerologie, Vivantes-Klinikum Berlin Süd, Berlin, Germany

Background The dramatic development of antimicrobial resistance in Neisseria gonorrhoeae is a serious problem for treatment and control of gonorrhoea. Numerous formerly effective therapeutic agents are no longer appropriable.

High level penicillin resistance and quinolone resistance disseminated globally. The third generation cephalosporins are amongst the last agents to remain effective. Reduced susceptibility to these cephalosporins is increasingly common. Currently the emergence of cephalosporine resistance in gonococci can be observed. There is a grave concern that multidrug resistant Neisseria gonorrhoeae strains will develop (MDR-NG)

Methods Non-selected collection of Neisseria gonorrhoeae isolates from all regions of Germany.

It is the aim of this surveillance to monitor the antimicrobial resistance to a range of therapeutically relevant antimicrobials as third generation cephalosporins (cefixime, ceftriaxone), azithromycin, gentamicin, spectinomycin, ciprofloxacin and penicillin.

Results We report current results from the 2011/12 antimicrobial resistance (AMR) surveillance in Germany.

More than $10 \%$ of the isolates displayed decreased susceptibility to cephalosporins, mostly to cefixime. Nearly $5 \%$ of all isolates showed a decreased susceptibility to both cephalosporins. All of these isolates also displayed resistance to ciprofloxacin.

Conclusion Cefixime is no longer recommended as first line therapy agent due to a high number of isolates with decreased susceptibility. Ceftriaxone is an appropriate treatment for gonorrhoea, but a drift to decreased susceptibility can be observed. Azithromycin remains still effective. Rates of ciprofloxacin and penicillin resistance are high across Germany. 